\title{
Perfect factorisations of bipartite graphs and Latin squares without proper subrectangles
}

\author{
I. M. Wanless \\ Department of Mathematics and Statistics \\ University of Melbourne \\ Parkville Vic 3052 Australia \\ ianw@ms.unimelb.edu.au \\ Submitted: November 16, 1998; Accepted January 22, 1999. \\ AMS Classifications: 05B15, 05C70.
}

\begin{abstract}
A Latin square is pan-Hamiltonian if every pair of rows forms a single cycle. Such squares are related to perfect 1-factorisations of the complete bipartite graph. A square is atomic if every conjugate is pan-Hamiltonian. These squares are indivisible in a strong sense - they have no proper subrectangles. We give some existence results and a catalogue for small orders. In the process we identify all the perfect 1-factorisations of $K_{n, n}$ for $n \leq 9$, and count the Latin squares of order 9 without proper subsquares.
\end{abstract}

\section{$\S 1$. Introduction}

For $k \leq n$, a $k \times n$ Latin rectangle is a $k \times n$ matrix of entries chosen from some set of symbols of cardinality $n$, such that no symbol is duplicated within any row or any column. Typically we assume that the symbol set is $\{1,2, \ldots, n\}$. We use $L(k, n)$ for the set of $k \times n$ Latin rectangles. Elements of $L(n, n)$ are called Latin squares of order $n$. The symbol in row $r$, column $c$ of a Latin rectangle $R$ is denoted by $R_{r c}$. A Latin square $S$ is idempotent if $S_{i i}=i$ for each $i$.

If the symbol set of a Latin rectangle $R$ is $\{1,2, \ldots, n\}$ then each row $r$ is the image of some permutation $\sigma_{r}$ of that set. That is, $R_{r i}=\sigma_{r}(i)$. Moreover, each pair of rows $(r, s)$ defines a permutation by $\sigma_{r, s}=\sigma_{r} \sigma_{s}^{-1}$. Naturally $\sigma_{r, s}=\sigma_{s, r}^{-1}$. Any of these permutations may be written as a product of disjoint cycles in the standard way. If this product consists of a single factor we call the permutation a full cycle permutation.

A subrectangle of a Latin rectangle is a submatrix (not necessarily consisting of adjacent entries) which is itself a Latin rectangle. If it happens to be a Latin square it is called a subsquare. An $a \times b$ subrectangle of a $k \times n$ Latin rectangle is proper provided we have the strict inequalities $1<a<k$ and $1<b<n$. A Latin square without subsquares 
of order 2 is said to be $N_{2}$ and a Latin square without proper subsquares is $N_{\infty}$. Latin squares with no proper subrectangles will be of central interest in this paper.

There are some important equivalence relations for Latin squares. Two squares are isotopic if one can be obtained from the other by rearranging the rows, rearranging the columns and renaming the symbols. The set of all squares isotopic to a given square forms an isotopy class. The second operation is conjugacy. Here instead of permuting within the sets of rows, columns and symbols, we permute the sets themselves. For example, starting with a square $S$, we might interchange rows with columns to get $S^{T}$, the transpose of $S$. Alternatively, we could interchange the roles of columns and symbols to get a square $R^{-1}(S)$, which we call the row inverse of $S$. Note that $R^{-1}(S)$ can be obtained from $S$ by replacing $\sigma_{r}$ by $\sigma_{r}^{-1}$ in each row $r$. If it happens that $S=R^{-1}(S)$ then clearly each $\sigma_{r}$ must be an involution and we say that $S$ is involutory. The operations of transposition and row inverse generate a conjugacy class consisting of 6 conjugates $\left\{S, S^{T}, R^{-1}(S),\left(R^{-1}(S)\right)^{T}, R^{-1}\left(S^{T}\right),\left(R^{-1}\left(S^{T}\right)\right)^{T}\right\}$. The closure of an isotopy class under conjugacy yields a main class.

Two edges of a graph are independent if they do not share a common vertex. A set of pairwise independent edges which covers the vertices of a graph is called a 1-factor (also known as a perfect matching). A partitioning of the edges of a graph into 1-factors is a 1-factorisation. A 1-factorisation is perfect if the union of any two of its 1-factors is a single (Hamiltonian) cycle. For a full discussion of 1-factorisations see [14], and for a short summary of known results consult [1].

There is a close relationship between Latin rectangles and 1-factorisations in regular bipartite graphs, in which each row of a rectangle corresponds to a 1-factor. For each $R \in L(k, n)$ we can form $G(R)$, a $k$-regular subgraph of $K_{n, n}$ in which the vertex sets correspond to the columns and the symbols, and an edge indicates that the symbol is used in the column. The Latin property of $R$ means that the edges corresponding to the (column, symbol) pairs within a row are a 1-factor, and the 1-factors corresponding to different rows are disjoint. Hence $R$ prescribes a 1-factorisation of $G(R)$ in a natural way. In this paper we investigate the case where the 1-factorisation happens to be perfect. An alternative way to view our results in terms of transversal designs will be discussed briefly in $\S 5$.

If $R$ is a $2 \times a$ subrectangle of some Latin square $L$, and $R$ is minimal in that it contains no $2 \times b$ subrectangle for $b<a$, then we say that $R$ is a row cycle of length $a$. Column cycles and symbol cycles are defined similarly, and the operations of conjugacy on $L$ interchange these objects. Note that there is a natural 1:1 length-preserving correspondence between row cycles involving rows $r, s$ and cycles in $\sigma_{r, s}$. We are interested in the case where all row cycles are as long as possible: 
Definition. A Latin rectangle $R \in L(k, n)$ is pan-Hamiltonian if every row cycle of $R$ has length $n$. Equivalently, $R$ is pan-Hamiltonian if $\sigma_{r, s}$ is a full cycle permutation for each pair of rows $r, s$ in $R$.

The name pan-Hamiltonian comes from [9], where it is used to describe a Latin square in which each symbol cycle is Hamiltonian. We prefer to base our definition on row cycles because it then makes sense for Latin rectangles which are not squares. Our definition is clearly related to that of [9] by conjugacy.

\section{$\S 2 . \quad$ Basic properties}

We examine a few simple properties of pan-Hamiltonian squares. Firstly, from the discussion in the introduction we have:

Lemma 1. There is a pan-Hamiltonian square of order $n$ if and only if $K_{n, n}$ has a perfect 1-factorisation.

In fact the concepts of pan-Hamiltonian squares and bipartite perfect 1-factorisations are so closely linked that in what follows we will sometimes consider them synonymous. Our second result provides further motivation for our study.

Lemma 2. A Latin square is pan-Hamiltonian if and only if it contains no proper subrectangles. In particular every pan-Hamiltonian square is $N_{\infty}$.

Proof: If $L \in L(n, n)$ is not pan-Hamiltonian then it contains a row cycle of length less than $n$ and this row cycle immediately gives a proper subrectangle. Conversely, suppose $L$ contains a proper subrectangle $R$. Then $R$ has at least two rows so it contains at least one row cycle $C$. For $R$ to be proper, $C$ must have length less than $n$. But $C$ is a row cycle of $L$, so $L$ is not pan-Hamiltonian.

Lemma 2 gives a good reason to be interested in pan-Hamiltonian Latin squares, but also shows that constructing them is likely to be difficult. Note that at this stage the existence question for $N_{\infty}$ squares is not completely resolved. Heinrich [8] gave a construction for $n=p q \neq 6$ where $p$ and $q$ are prime, which was later generalised in [2] to all orders except those of the form $2^{a} 3^{b}$. Only a few sporadic orders from the remaining case have since been settled. Some small order examples have been discovered by computer searches. Perfect 1-factorisations have also been successfully employed and offer greater hope of producing infinite classes of examples. 
Lemma 3. If $L$ is a pan-Hamiltonian square then so is any square isotopic to $L$, and so is $R^{-1}(L)$.

Proof: Isotopies preserve the lengths of row cycles and hence also the pan-Hamiltonian property. The implication that $R^{-1}(L)$ is pan-Hamiltonian follows from the observation that the inverse of a full cycle permutation is also a full cycle permutation.

Note that the operations discussed in Lemma 3 correspond to the natural notion of isomorphism for perfect 1-factorisations. Suppose that from a Latin square $L$ we construct a complete bipartite graph $G$ with vertex sets $U$ and $V$, and a 1 -factorisation of $G$ with 1-factors $F$. Then an isotopy of $L$ corresponds to a relabelling/reordering of the sets $U, V$ and $F$. Also, taking the row inverse of $L$ corresponds to switching the sets $U$ and $V$. For a formal definition of isomorphism between 1-factorisations, and some invariants with which to distinguish non-isomorphic factorisations, see Chapter 11 of [14]. One of the ideas there is that of a train, which we now define for factorisations of complete bipartite graphs.

Suppose we have $G$, a copy of $K_{n, n}$ in which the vertex sets are $U$ and $V$. We define $\mathcal{T}(\mathcal{F})$, the train of a 1 -factorisation $\mathcal{F}$ of $G$, to be a directed graph (with loops) on the triples in $U \times V \times \mathcal{F}$. Each of the $n^{2}(n-1)$ vertices has outdegree 1. The edge from $[u, v, f]$ goes to $\left[u^{\prime}, v^{\prime}, f^{\prime}\right]$ where $f$ contains the edges $u v^{\prime}$ and $u^{\prime} v$ and $f^{\prime}$ contains the edge $u v$. It should be clear that two isomorphic factorisations of $G$ must have isomorphic trains.

It is worth pointing out that although the factorisations corresponding to $L$ and $R^{-1}(L)$ are isomorphic, the squares $L$ and $R^{-1}(L)$ may or may not be isotopic. Examples of both types will be given in $\S 6$.

A particularly simple Latin square on the set $\{1,2, \ldots, n\}$ is the Cayley table $\mathcal{C}_{n}$ of the cyclic group of order $n$. Here $\mathcal{C}=\mathcal{C}_{n}$ is defined by $\mathcal{C}_{i j} \equiv i+j-1(\bmod n)$. By symmetry all row cycles in $\mathcal{C}_{n}$ have length dividing $n$. If $n$ happens to be prime the row cycles must be of length $n$, so $\mathcal{C}_{n}$ will be a pan-Hamiltonian Latin square. Hence

Lemma 4. Perfect 1-factorisations of $K_{p, p}$ exist for all prime $p$.

In the next section we strengthen Lemma 4 , by constructing non-isomorphic perfect 1-factorisations of $K_{p, p}$ from a perfect 1-factorisation of $K_{p+1}$.

For any Latin square $L$, define $\nu(L)$ to be the number of conjugates of $L$ which are pan-Hamiltonian. Note that $\nu(\cdot)$ is a main class invariant as a consequence of Lemma 3 , so we can sensibly write $\nu(M)$ for a main class $M$. An interesting property of $\mathcal{C}_{n}$ is that it is isotopic to all of its conjugates (Theorem 4.2 .2 of [5]). So by Lemma $3, \nu\left(\mathcal{C}_{p}\right)=6$ for prime $p$. In other words, every square in the main class of $\mathcal{C}_{p}$ is pan-Hamiltonian. In general this is not true of main classes containing pan-Hamiltonian squares. As we shall see in $\S 6$ below, all Latin squares $L$ of order at most 9 (other than those isotopic to $\mathcal{C}_{p}$ for 
some prime $p$ ) have $\nu(L)$ either 0 or 2. Note that $\nu(L) \in\{0,2,4,6\}$ by Lemma 3. All of these values are achievable. In $\S 4$ we will look at squares for which $\nu(\cdot)=6$. To find a square for which $\nu(\cdot)=4$ it is useful to consider possible symmetries of the square:

Lemma 5. Suppose $L$ is a pan-Hamiltonian square. If $L$ is isotopic to $R^{-1}(L)$ then $\nu(L) \in\{2,6\}$. Alternatively, if $L$ is isotopic to $L^{T}$ then $\nu(L) \in\{4,6\}$.

Proof: We write $A \sim B$ if both $A$ and $B$ are pan-Hamiltonian, or neither is. Let the conjugates of $L$ be $L_{1}=L, L_{2}=L^{T}, L_{3}=R^{-1}(L), L_{4}=\left(R^{-1}(L)\right)^{T}, L_{5}=R^{-1}\left(L^{T}\right)$, $L_{6}=\left(R^{-1}\left(L^{T}\right)\right)^{T}$. Note that $L_{1}$ is pan-Hamiltonian by assumption. We make use of Lemma 3, starting with the observation that $L_{1} \sim L_{3}, L_{2} \sim L_{5}$ and $L_{4} \sim L_{6}$. If $L_{1}$ and $L_{3}$ are isotopic then $L_{2} \sim L_{4}$ and $L_{5} \sim L_{6}$ because they are also isotopic pairs. But then $L_{2} \sim L_{4} \sim L_{5} \sim L_{6}$, from which the first assertion of the lemma follows. A similar argument works if $L_{1} \sim L_{2}$. In this case $L_{3} \sim L_{5}$ and $L_{4} \sim L_{6}$, so that $L_{1} \sim L_{2} \sim L_{3} \sim L_{5}$ and at least four conjugates are pan-Hamiltonian.

As an application of Lemma 5, we will meet pan-Hamiltonian squares in the next section which are derived from perfect 1-factorisations of complete graphs. These squares are involutory, so they always have $\nu(\cdot) \in\{2,6\}$. The other part of Lemma 5 is more promising for finding examples of squares for which $\nu(\cdot)=4$. Indeed such a square is given in (1). This square is symmetric about its main diagonal so it is sufficient to note that it is pan-Hamiltonian but that the symbols 1 and 11 form three separate symbol cycles.

$$
\left(\begin{array}{ccccccccccc}
1 & 2 & 3 & 4 & 5 & 6 & 7 & 8 & 9 & 10 & 11 \\
2 & 3 & 4 & 5 & 6 & 7 & 8 & 9 & 10 & 11 & 1 \\
3 & 4 & 5 & 6 & 11 & 8 & 1 & 10 & 7 & 9 & 2 \\
4 & 5 & 6 & 7 & 9 & 2 & 11 & 1 & 8 & 3 & 10 \\
5 & 6 & 11 & 9 & 4 & 1 & 10 & 7 & 3 & 2 & 8 \\
6 & 7 & 8 & 2 & 1 & 10 & 3 & 11 & 5 & 4 & 9 \\
7 & 8 & 1 & 11 & 10 & 3 & 9 & 4 & 2 & 6 & 5 \\
8 & 9 & 10 & 1 & 7 & 11 & 4 & 2 & 6 & 5 & 3 \\
9 & 10 & 7 & 8 & 3 & 5 & 2 & 6 & 11 & 1 & 4 \\
10 & 11 & 9 & 3 & 2 & 4 & 6 & 5 & 1 & 8 & 7 \\
11 & 1 & 2 & 10 & 8 & 9 & 5 & 3 & 4 & 7 & 6
\end{array}\right)
$$

In Lemma 4 we saw an existence result for pan-Hamiltonian Latin squares of some orders. Now we look at some orders for which these squares do not exist.

Lemma 6. If $R \in L(k, n)$ is pan-Hamiltonian then either $n$ is odd or $k \leq 2$.

Proof: Suppose $n$ is even and that $r, s$ are two rows of $R$. By definition, $\sigma_{r, s}$ is a full cycle permutation on an even number of symbols. In particular $\sigma_{r, s}$ is an odd permutation, so $\sigma_{r}$ and $\sigma_{s}$ must be of different parities. As this is true for any pair of rows in $R$, we see that there cannot be more than 2 rows. 
The electronic journal of Combinatorics 6 (1999), \#R9

Corollary. Up to isomorphism, $\mathcal{C}_{2}$ is the only pan-Hamiltonian Latin square of even order.

Gibbons and Mendelsohn [7] attempted to find a pan-Hamiltonian square of order 12, because they knew such a square would be $N_{\infty}$ (see Lemma 2). Lemma 6 explains why their search failed, and also rules out using pan-Hamiltonian squares to completely settle the remaining existence questions for $N_{\infty}$ squares. The best we can hope for is to find examples for the orders which are powers of three. This has been achieved [6] for sporadic orders including $3^{a}$ for $a \leq 5$, by the techniques discussed in the next section.

\section{$\S 3 . \quad$ Factorisations of complete graphs}

In this section we examine connections between perfect 1-factorisations of complete graphs and those in complete bipartite graphs.

In [6, pg116] a construction is provided for an $N_{\infty}$ square of order $\mathrm{n}$, given a perfect 1-factorisation of the complete graph $K_{n+1}$ (which can only be found if $n$ is odd). This construction, also given in [14] and mentioned in [2], is attributed to "A. Rosa and others". The construction we give is related by conjugacy.

Suppose we have a factorisation $\mathcal{F}$ of $K_{n+1}$ consisting of 1 -factors $F_{1}, F_{2}, \ldots, F_{n}$. Let the vertices of the $K_{n+1}$ be $v_{1}, v_{2}, \ldots, v_{n+1}$ and rename the 1 -factors if necessary so that $F_{i}$ contains the edge $v_{i} v_{n+1}$. We construct a Latin square $S(\mathcal{F})$ in which row $i$ is determined from $F_{i}$ as follows. The row permutation $\sigma_{i}$ is the product of $\frac{1}{2}(n-1)$ disjoint 2-cycles, where there is a 2-cycle $(a b)$ corresponding to each edge $v_{a} v_{b} \in F_{i} \backslash\left\{v_{i} v_{n+1}\right\}$. Since the factors $\left\{F_{i}\right\}$ are pairwise disjoint by definition, $S(\mathcal{F})$ is a Latin square. Also, by construction $S(\mathcal{F})$ is involutory and idempotent. Finally, note that the row cycles involving rows $i$ and $j$ of $S(\mathcal{F})$ correspond in a natural way to cycles in $F_{i} \cup F_{j}$. In particular if $\mathcal{F}$ happens to be a perfect 1-factorisation then $S(\mathcal{F})$ is pan-Hamiltonian. We have:

Lemma 7. The map $\mathcal{F} \rightarrow S(\mathcal{F})$ is a bijection between 1-factorisations of $K_{n+1}$ (with fixed vertex labels $v_{1}, v_{2}, \ldots, v_{n+1}$ ) and idempotent involutory squares in $L(n, n)$. It maps perfect 1-factorisations to pan-Hamiltonian squares.

Proof: It suffices to show the construction of $S(\mathcal{F})$ is 'reversible'. So suppose that $L$ is an idempotent involutory square of order $n$. Then in $L$, each row permutation $\sigma_{i}$ is an involution. Since $L$ is idempotent each $\sigma_{i}$ fixes $i$ and it follows that $\sigma_{i}$ cannot fix any $j \neq i$ without breaching the Latin property of $L$. Hence each $\sigma_{i}$ must be a product of $\frac{1}{2}(n-1)$ disjoint 2-cycles. It is a simple matter now to construct the factorisation of $K_{n+1}$ for which $L$ is the image, by using edges corresponding to these 2 -cycles. 
Corollary. If $K_{n+1}$ has a perfect 1-factorisation, then so does $K_{n, n}$.

The converse of this last result is not true. Note that $K_{3}$ does not even have a 1-factor. However $K_{2,2}$ has a perfect 1-factorisation (cf. the corollary to Lemma 6 ). It may well be that this (somewhat trivial) case is the only exception. This would follow, if the following widely believed conjecture were proved.

Conjecture. $K_{n}$ has a perfect 1-factorisation for all even positive integers $n$.

If there is a counterexample, then by [14, p.127] it has $n>50$. Note that Wallis chooses to exclude the trivial case $n=2$. However we see no reason to do this given that the edge in $K_{2}$ is a 1-factorisation and (vacuously) any two 1-factors in this factorisation form a Hamiltonian cycle.

Given the preceding comments, plus the Corollary to Lemma 7, it seems reasonable to suggest that:

Conjecture. $K_{n, n}$ has a perfect 1 -factorisation for $n=2$ and all odd positive integers $n$.

Again, any counterexample must have $n>50$. We look next at some of the constructions which justify this claim.

Suppose $n$ is odd. We define a 1 -factorisation $E_{n+1}$ of $K_{n+1}$. There is one special vertex labelled $\infty$, the other vertices are labelled with the congruence classes of integers modulo $n$. For $i=1,2, \ldots, n$, define factor $f_{i}$ to consist of the edges $(i-1)(i+1)$, $(i-2)(i+2), \ldots,\left(i-\left\lfloor\frac{1}{2} n\right\rfloor\right)\left(i+\left\lfloor\frac{1}{2} n\right\rfloor\right)$ together with $i \infty$. Whenever $n$ is prime the resulting 1-factorisation $E_{n+1}$ is perfect [14]. Hence by employing the corollary to Lemma 7 we have an alternate proof of Lemma 4 . In fact we can squeeze out a stronger result.

Lemma 8. Non-isomorphic perfect 1-factorisations of $K_{p, p}$ exist for all prime $p \geq 7$.

Proof: The construction which is the basis for Lemma 7 is not robust in the following sense. Given two isomorphic perfect 1-factorisations $F_{1}$ and $F_{2}$ of $K_{n+1}$ it is possible that the perfect 1-factorisations of $K_{n, n}$ given by $S\left(F_{1}\right)$ and $S\left(F_{2}\right)$ will not be isomorphic. This is the case only because of the special role played by the vertex labelled $v_{n+1}$ in the map $\mathcal{F} \rightarrow S(\mathcal{F})$. In $E_{n+1}$ there are (up to symmetry) just two choices for $v_{n+1}$ : either $v_{n+1}=\infty$ or without loss of generality $v_{n+1}=1$. To prove the lemma we show that these two choices give non-isomorphic results. To do this it is sufficient to show that they produce non-isomorphic trains, as defined in $\S 2$. For the remainder of this proof all calculations will be performed modulo $n$.

In the first instance let $v_{i}=i$ for $i=1, \ldots, n$ and put $v_{n+1}=\infty$. It is easy to see that the resulting pan-Hamiltonian square $S$ is defined by $S_{i j} \equiv 2 i-j$, and hence is isotopic to $\mathcal{C}_{n}$. Let $h=2^{-1} \equiv \frac{1}{2}(n+1)$. For any vertex $[u, v, f]$ of $\mathcal{T}(S)$ there is an edge to $[u, v, f]$ 
from $\left[h u-h v+f,-h u+h v+f, h^{2} u+h^{2} v+h f\right]$. It follows that $\mathcal{T}(S)$ is 1-regular, since we know every vertex has outdegree 1.

Now suppose that we had swapped the labels on $v_{n+1}$ and $v_{1}$ before calculating a pan-Hamiltonian square $S^{\prime}$. The definition of $S^{\prime}$ is easy enough:

$$
S_{i j}^{\prime} \equiv \begin{cases}i & \text { if } i=j \\ 1 & \text { if } i \equiv 2 j-1 \\ \frac{1}{2}(i+1+(i-1) n) & \text { if } j=1 \\ i-j+1 & \text { otherwise. }\end{cases}
$$

We claim that in $\mathcal{T}\left(S^{\prime}\right)$ the vertex $[2,3,1]$ has indegree at least 2 , assuming $n \geq 7$. In fact the edges from both $[n, 2,2]$ and $\left[\frac{1}{2}(n+1), \frac{1}{2}(n+3), \frac{1}{2}(n+5)\right]$ terminate at $[2,3,1]$ in this case. Hence the trains $\mathcal{T}(S)$ and $\mathcal{T}\left(S^{\prime}\right)$ cannot be isomorphic, and we have two essentially different squares as claimed.

Apart from $E_{p+1}$ for prime $p$, there is only one infinite family of perfect 1-factorisations of complete graphs known. For prime $p$ the idea is to consider $K_{2 p}$ as the union of two graphs: $K_{p, p}$ and a double copy of $K_{p}$. A 1-factorisation is then built up from 1factorisations of the two parts. For exact details the interested reader should consult [1] or [14]. We mention the result for two reasons. Firstly, the method demonstrates further connections between perfect 1-factorisations of complete bipartite graphs and complete graphs. Secondly of course, it gives the following existence result, via the corollary to Lemma 7.

Lemma 9. If $p$ is prime then $K_{2 p-1,2 p-1}$ has a perfect 1-factorisation.

In this section we have looked for perfect 1-factorisations of $K_{n, n}$ which are derived from perfect 1-factorisations of $K_{n+1}$. As a footnote we observe that in general there are plenty of perfect 1-factorisations of $K_{n, n}$ which do not correspond in any obvious way to a perfect 1-factorisation of $K_{n+1}$. We shall see in $\S 6$ that up to isomorphism there are 37 perfect 1-factorisations of $K_{9,9}$ and only one of $K_{10}$.

\section{$\S 4$. Atomic squares}

In his review [13] of [8], Stein discusses Latin squares with an indivisibility property stronger than $N_{\infty}$. In our language, Stein's squares are those $S$ for which neither $S$ nor $S^{T}$ has a proper subrectangle. Perhaps a more natural concept is obtained by not favouring rows and columns over symbols. Hence, 
Definition. A Latin square is atomic if none of its conjugates has a proper subrectangle.

The example (1) confirms that the atomic squares are a strict subset of Stein's squares. Of course we use the name 'atomic' here in the classical sense, meaning 'indivisible'. We have the following characterisation.

Lemma 10. A Latin square $L$ is atomic if and only if $\nu(L)=6$. To test whether $L$ is atomic it suffices to establish that $L, L^{T}$ and $\left(R^{-1}(L)\right)^{T}$ are pan-Hamiltonian.

Proof: By Lemma 3 the three listed conjugates are pan-Hamiltonian if and only if all six conjugates of $L$ are pan-Hamiltonian. The remainder of the lemma is a straightforward application of Lemma 2.

Stein's main question in [13] is one of existence. We are now in a position to partly answer his query. Firstly, when $p$ is prime we know by Lemma 10 that $\mathcal{C}_{p}$ is atomic since $\nu\left(\mathcal{C}_{p}\right)=6$. This much was alluded to by Stein [13]. However, by combining Lemma 10 with Lemma 6 we also know that atomic squares of even composite order do not exist.

So far the only examples of atomic squares we have seen are the family of $\mathcal{C}_{p}$ for $p$ prime. To show that the class is broader, we now display a second infinite(?) family, although this family also consists only of prime orders.

Lemma 11. Let $p \geq 11$ be a prime. If 2 is a primitive root modulo $p$ then there exists an atomic square of order $p$ outside the main class of $\mathcal{C}_{p}$.

Proof: We show that the non-isomorphic 1-factorisations exhibited in Lemma 8 both lead to atomic squares. The first of these 1 -factorisations gave a square isotopic to $\mathcal{C}_{p}$, so we need only examine the second. By Lemma 5 it suffices to show that the transpose of the square $S^{\prime}$ defined by setting $n=p$ in (2) is pan-Hamiltonian. Let $M$ be the Latin square in which $M_{i j} \equiv i-j+1(\bmod p)$, so that $S^{\prime}$ is nearly a copy of $M$. We need to show that any two columns $a$ and $b$ of $S^{\prime}$ consist of a single column cycle; something we know is true in $M$ because $M$ is isotopic to $\mathcal{C}_{p}$. We split into two cases.

Case 1. $1<a<b$

Consider 2-regular bipartite graphs with vertices $r_{1}, \ldots, r_{p}$ and $s_{1}, \ldots, s_{p}$ corresponding to the rows and symbols respectively. When such a graph is made from the entries in columns $a$ and $b$ of $M$, let the graph be called $G$ and when the columns $a$ and $b$ of $S^{\prime}$ are used, let the graph be $G^{\prime}$. Then $G^{\prime}$ is obtained from $G$ by removing four edges $r_{a} s_{1}$, $r_{2 a-1} s_{a}, r_{b} s_{1}$ and $r_{2 b-1} s_{b}$, and replacing them with $r_{a} s_{a}, r_{2 a-1} s_{1}, r_{b} s_{b}$ and $r_{2 b-1} s_{1}$. As already noted, $G$ must be a single cycle. Orient it so that as we traverse it clockwise we encounter in order $r_{a}, s_{1}$ then $r_{b}$. We next establish the order in which the crucial edges 
$r_{2 a-1} s_{a}$ and $r_{2 b-1} s_{b}$ will be encountered as we traverse $G$ clockwise from $r_{b}$. By the symmetry inherent in $M$ we know that the clockwise distance from $r_{i}$ to $s_{i}$ around $G$ cannot depend on $i$. It follows that on our transversal we cannot encounter $r_{b}, s_{b}, s_{a}, r_{a}$ in that order, so we must reach the edge $r_{2 a-1} s_{a}$ before $r_{2 b-1} s_{b}$. Similarly the orientation of the edge $r_{a} s_{1}$ determines that we must reach $r_{2 a-1}$ before $s_{a}$ and the orientation of the edge $r_{b} s_{1}$ determines that we must reach $s_{b}$ before $r_{2 b-1}$. In short, we must have the situation depicted in Figure 1(a). It is then clear (see Figure 1(b)) that $G^{\prime}$ is also a single cycle.

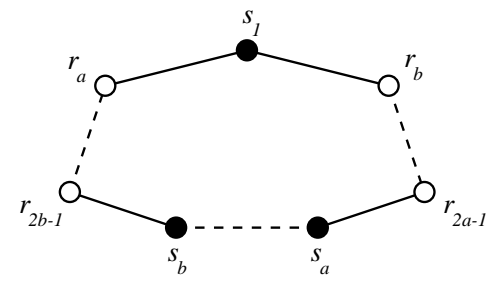

Figure 1(a): $G$ in case 1

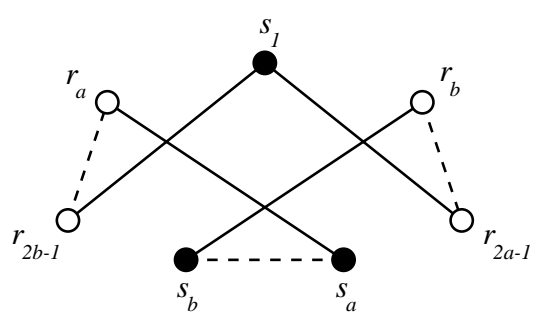

Figure 1(b): $G^{\prime}$ in case 1

Case 2. $1=a<b$

This time we let $G$ be the graph formed by the union of the first column of $S^{\prime}$ with column $b$ of $M$, while $G^{\prime}$ comes from columns 1 and $b$ of $S^{\prime}$. Note that $G^{\prime}$ can be obtained from $G$ by deleting the edges $r_{b} s_{1}$ and $r_{2 b-1} s_{b}$ and replacing them with $r_{b} s_{b}$ and $r_{2 b-1} s_{1}$.

Consider a function $f$ from the symbol set to itself which maps $S_{i 1}^{\prime}$ to $S_{i b}^{\prime}$ for each $i$. Then $f(x) \equiv 2 x-b(\bmod p)$. Let $f^{m}$ be $f$ composed with itself $m$ times, so that $f^{m}(x) \equiv 2^{m} x-\left(2^{m}-1\right) b$. We look for fixed points of $f^{m}$. Note that $f^{m}(x)=x$ if and only if $\left(2^{m}-1\right)(x-b) \equiv 0(\bmod p)$. Here is where we use the assumption that 2 is primitive modulo $p$ and hence $2^{m}-1 \equiv 0(\bmod p)$ only if $p-1 \mid m$. Translating this knowledge, we see that our graph $G$ consists of precisely two cycles, one of which is a digon on the vertices $r_{2 b-1}$ and $s_{b}$. It is then obvious (Figure 2) that the surgery performed to create $G^{\prime}$ from $G$ cannot fail to create a single cycle.

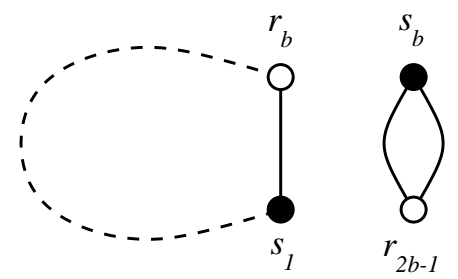

Figure 2(a): $G$ in case 2

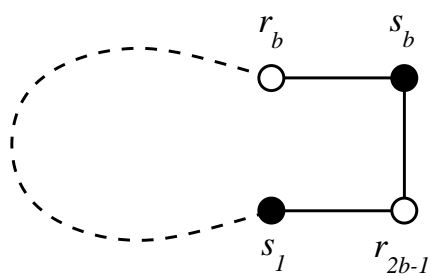

Figure 2(b): $G^{\prime}$ in case 2 
With regard to this last result, it is of interest to note that in the cases when 2 is not a primitive root modulo $p$, the graph $G$ in Figure 2(a) consists of at least 3 cycles and hence $G^{\prime}$ cannot be a single cycle. It follows that our construction never gives an atomic square in such a case. However it does give a square which is nearly atomic. All row cycles are Hamiltonian and only column cycles involving the first column fail to be Hamiltonian (a similar statement holds for the symbol cycles).

We can use Lemma 10 to screen our catalogue in $\S 6$ for atomic squares. The conclusion is that there are no atomic squares of order below 11 except those arising from groups of prime order. Note that Lemma 11 allows us to construct an atomic square of order 11 different from $\mathcal{C}_{11}$.

Finally we note that atomic squares of composite orders do exist. For example, it is possible to create an atomic square of order 27 by applying the process described in $\S 3$ to the perfect 1-factorisation of $K_{28}$ described in [3]. It would be of some interest to find an atomic square of an order which is not a prime power. No such square is known to the author.

\section{$\S 5$. Transversal designs}

A number of our results can be neatly expressed in terms of transversal designs (thanks to the referee for making this observation). A transversal design $T D(n, k)$ is a set of $n k$ points partitioned into $k$ groups of $n$ elements each, together with a set of blocks. Each block must contain exactly one point from each group and any two points from different groups must occur in exactly one block. It is well known that Latin squares can be formed from a $T D(n, 3)$. Simply choose a correspondence from the three groups, in some order, to the rows, columns and symbols; then each of the $n^{2}$ blocks designates a symbol to be placed in a particular row and column. Any two squares derived in this manner from the same transversal design will be in the same main class. More generally, a $T D(n, k)$ is really just a set of $k-2$ mutually orthogonal Latin squares by another name [4].

Suppose we are given $T$, a $T D(n, 3)$ with groups $G_{1}, G_{2}$ and $G_{3}$. For each point $p \in G_{1}$ there is a 1-factor between $G_{2}$ and $G_{3}$ given by the restriction to $G_{2} \cup G_{3}$ of all the blocks containing $p$. Similarly, if two points $p_{1}, p_{2} \in G_{1}$ are chosen, then they induce a 2-factor on $G_{2} \cup G_{3}$. If this 2-factor is always a Hamiltonian cycle regardless of the choice of $p_{1}, p_{2}$ then $T$ corresponds to a pan-Hamiltonian Latin square (provided $G_{1}$ is chosen to represent the rows). We might say that $T$ is pan-Hamiltonian with respect to $G_{1}$. Of course, if $T$ is pan-Hamiltonian with respect to all of its groups then $T$ corresponds to an atomic square. In this case we say that $T$ is an atomic transversal design (ATD).

Translating earlier results into the new terminology we find that an $A T D(n, 3)$ cannot exist if $n>2$ is even (Lemma 6), but that there is an $A T D(p, 3)$ for all prime $p$ (Lemma 4). 
The electronic journal of Combinatorics 6 (1999), \#R9

Indeed we can construct non-isomorphic $A T D(p, 3)$ for many primes $p$ (Lemma 11). We also know that an $A T D(27,3)$ exists but, as we will discover in the next section, an $A T D(9,3)$ does not.

One bonus from choosing the transversal design formulation is that it allows an immediate generalisation. We can define a $T D(n, k)$ to be an $A T D(n, k)$ if its projection onto any 3 of its groups yields an $\operatorname{ATD}(n, 3)$. However, the only such designs known at this stage for $k>3$ arise from the Desarguesian projective planes of prime order $p$. Such planes yield an $A T D(p, p+1)$ in which every restriction to 3 groups is atomic because it is isomorphic to $\mathcal{C}_{p}$. Hence we have a generalisation of Lemma 4 .

\section{$\S 6 . \quad$ Small orders}

For $n \in\{2,3,5\}$ the catalogue of Latin squares in [5] shows that there is a single main class of $N_{\infty}$ square of order $n$, namely that of $\mathcal{C}_{n}$. According to Norton [11] (notwithstanding the later correction by Sade [12]) there are precisely two main classes of $N_{2}$ squares of order 7 . Both turn out to be $N_{\infty}$ and to contain pan-Hamiltonian squares. An example from the main class other than that of $\mathcal{C}_{7}$ is:

$$
A_{7}=\left(\begin{array}{lllllll}
1 & 2 & 3 & 4 & 5 & 6 & 7 \\
2 & 3 & 4 & 5 & 6 & 7 & 1 \\
3 & 4 & 5 & 6 & 7 & 1 & 2 \\
4 & 6 & 7 & 2 & 1 & 3 & 5 \\
5 & 7 & 2 & 1 & 3 & 4 & 6 \\
6 & 5 & 1 & 7 & 4 & 2 & 3 \\
7 & 1 & 6 & 3 & 2 & 5 & 4
\end{array}\right)
$$

We note that $\nu\left(A_{7}\right)=2$ and that $A_{7}$ is isotopic to $R^{-1}\left(A_{7}\right)$.

There are 37 main classes $M$ containing pan-Hamiltonian Latin squares of order 9 . In each case $\nu(M)=2$. Two examples are:

$$
A_{9}=\left(\begin{array}{lllllllll}
1 & 2 & 3 & 4 & 5 & 6 & 7 & 8 & 9 \\
2 & 4 & 1 & 5 & 7 & 9 & 8 & 6 & 3 \\
3 & 8 & 9 & 2 & 4 & 5 & 6 & 1 & 7 \\
4 & 9 & 2 & 3 & 1 & 8 & 5 & 7 & 6 \\
5 & 3 & 8 & 1 & 6 & 7 & 9 & 4 & 2 \\
6 & 5 & 7 & 8 & 9 & 4 & 2 & 3 & 1 \\
7 & 1 & 5 & 6 & 8 & 2 & 3 & 9 & 4 \\
8 & 7 & 6 & 9 & 3 & 1 & 4 & 2 & 5 \\
9 & 6 & 4 & 7 & 2 & 3 & 1 & 5 & 8
\end{array}\right), \quad B_{9}=\left(\begin{array}{lllllllll}
1 & 2 & 3 & 4 & 5 & 6 & 7 & 8 & 9 \\
2 & 4 & 1 & 9 & 3 & 8 & 6 & 5 & 7 \\
3 & 1 & 7 & 6 & 8 & 9 & 5 & 4 & 2 \\
4 & 3 & 5 & 7 & 1 & 2 & 8 & 9 & 6 \\
5 & 8 & 2 & 1 & 9 & 7 & 4 & 6 & 3 \\
6 & 9 & 4 & 8 & 7 & 5 & 3 & 2 & 1 \\
7 & 6 & 8 & 5 & 2 & 3 & 9 & 1 & 4 \\
8 & 7 & 9 & 2 & 6 & 4 & 1 & 3 & 5 \\
9 & 5 & 6 & 3 & 4 & 1 & 2 & 7 & 8
\end{array}\right) .
$$

We intend to give an entire catalogue, but the above format is impractically bulky. Instead, we describe a Latin square using a single line of text. We do this by first reducing 
the square (that is, putting its first row and column in natural order). Then since the first row and column are known, we omit them. For the other rows, we write the remaining entries out in order from left to right, but without spaces. We process the rows from top to bottom, placing a dot between rows. The first part of the list looks like this:

$41579863 \cdot 89245617 \cdot 92318576 \cdot 38167942 \cdot 57894231 \cdot 15682394 \cdot 76931425 \cdot 64723158$, $41963857 \cdot 82147965 \cdot 17629538 \cdot 94832671 \cdot 58794213 \cdot 65281394 \cdot 79315426 \cdot 36578142$, $41689537 \cdot 52197846 \cdot 86712395 \cdot 64873912 \cdot 17935428 \cdot 39248651 \cdot 95361274 \cdot 78524163$, $41785936 \cdot 52947168 \cdot 85372691 \cdot 67891423 \cdot 19523847 \cdot 38169254 \cdot 96214375 \cdot 74638512$, $41768935 \cdot 17924658 \cdot 98235176 \cdot 64879321 \cdot 79513842 \cdot 86392514 \cdot 35641297 \cdot 52187463$, $51689347 \cdot 19578624 \cdot 62817935 \cdot 97124863 \cdot 85743291 \cdot 36295418 \cdot 74932156 \cdot 48361572$, $41679358 \cdot 17824695 \cdot 56291837 \cdot 38947162 \cdot 85712943 \cdot 69583421 \cdot 94135276 \cdot 72368514$, $46587193 \cdot 14962578 \cdot 65329817 \cdot 87134962 \cdot 58793241 \cdot 39218456 \cdot 91675324 \cdot 72841635$, $41975836 \cdot 78629451 \cdot 92813675 \cdot 39764218 \cdot 54198327 \cdot 15382964 \cdot 67241593 \cdot 86537142$, $41835697 \cdot 62987541 \cdot 97368215 \cdot 74129863 \cdot 89513472 \cdot 15642938 \cdot 56791324 \cdot 38274156$.

The lines above represent ten pan-Hamiltonian Latin squares (one per line) in the condensed format described. Each of these ten squares is isotopic to its row inverse. In the other 27 main classes (listed below) there is no such symmetry. Note that $A_{9}, B_{9}$ in (4) are respectively the first square in the list above and the first square in the list below.

$41938657 \cdot 17689542 \cdot 35712896 \cdot 82197463 \cdot 94875321 \cdot 68523914 \cdot 79264135 \cdot 56341278$, $51679438 \cdot 75124896 \cdot 12895367 \cdot 68937142 \cdot 34582971 \cdot 96248513 \cdot 49713625 \cdot 87361254$, $41598376 \cdot 52879614 \cdot 97613258 \cdot 16947823 \cdot 39285147 \cdot 85362491 \cdot 64721935 \cdot 78134562$, $41895673 \cdot 69148257 \cdot 17389562 \cdot 84723196 \cdot 92574831 \cdot 35612948 \cdot 76931425 \cdot 58267314$, $41795638 \cdot 69271845 \cdot 17328956 \cdot 74983162 \cdot 82539417 \cdot 95864321 \cdot 56147293 \cdot 38612574$, $41567938 \cdot 95748216 \cdot 17395862 \cdot 64829173 \cdot 82973451 \cdot 38214695 \cdot 79631524 \cdot 56182347$, $51648973 \cdot 98167254 \cdot 62789531 \cdot 19274368 \cdot 74923815 \cdot 35812496 \cdot 46395127 \cdot 87531642$, $46379158 \cdot 18924567 \cdot 82795613 \cdot 69187432 \cdot 71238945 \cdot 35841296 \cdot 94562371 \cdot 57613824$, $46879135 \cdot 14792568 \cdot 85627913 \cdot 78963421 \cdot 39214857 \cdot 61538294 \cdot 92145376 \cdot 57381642$, $48569173 \cdot 19842567 \cdot 56971832 \cdot 67123948 \cdot 95387421 \cdot 34618295 \cdot 71294356 \cdot 82735614$, $51649837 \cdot 46875912 \cdot 62937158 \cdot 79182364 \cdot 87314295 \cdot 35298641 \cdot 94761523 \cdot 18523476$, $51897364 \cdot 76948152 \cdot 62719835 \cdot 38271496 \cdot 19534278 \cdot 84625913 \cdot 97362541 \cdot 45183627$, 


$$
\begin{aligned}
& 41695837 \cdot 59781264 \cdot 38529671 \cdot 87364912 \cdot 94172358 \cdot 12843596 \cdot 65917423 \cdot 76238145, \\
& 41567893 \cdot 54872961 \cdot 76985312 \cdot 68391274 \cdot 92138457 \cdot 15249638 \cdot 39714526 \cdot 87623145, \\
& 41983675 \cdot 95271468 \cdot 17825936 \cdot 89362147 \cdot 34597821 \cdot 58649213 \cdot 76134592 \cdot 62718354, \\
& 41763895 \cdot 69218457 \cdot 17829563 \cdot 76984321 \cdot 94572138 \cdot 82395614 \cdot 35641972 \cdot 58137246, \\
& 51384697 \cdot 48697521 \cdot 62738915 \cdot 37921846 \cdot 89513472 \cdot 16249358 \cdot 94175263 \cdot 75862134, \\
& 41637895 \cdot 17948652 \cdot 68571923 \cdot 92863174 \cdot 54189237 \cdot 39215468 \cdot 76392541 \cdot 85724316, \\
& 51789364 \cdot 86912547 \cdot 62831975 \cdot 37248691 \cdot 78395412 \cdot 19524836 \cdot 94167253 \cdot 45673128, \\
& 47935168 \cdot 18692574 \cdot 62319857 \cdot 84273916 \cdot 59847231 \cdot 91568423 \cdot 76124395 \cdot 35781642, \\
& 51748936 \cdot 85697142 \cdot 62931857 \cdot 49163278 \cdot 38275491 \cdot 14829365 \cdot 97514623 \cdot 76382514, \\
& 41739865 \cdot 78691524 \cdot 52978136 \cdot 86342971 \cdot 39517248 \cdot 15864392 \cdot 94123657 \cdot 67285413, \\
& 41739658 \cdot 84961572 \cdot 37815926 \cdot 69348217 \cdot 92173845 \cdot 15682394 \cdot 56297431 \cdot 78524163, \\
& 48963175 \cdot 17689542 \cdot 59712368 \cdot 86274913 \cdot 34897251 \cdot 62531894 \cdot 91345627 \cdot 75128436, \\
& 41583976 \cdot 86914257 \cdot 39725618 \cdot 64897123 \cdot 97248531 \cdot 15362894 \cdot 72139465 \cdot 58671342, \\
& 45967138 \cdot 16728594 \cdot 59613827 \cdot 97281643 \cdot 34879215 \cdot 68194352 \cdot 72345961 \cdot 81532476, \\
& 47639158 \cdot 14768592 \cdot 68971325 \cdot 39124867 \cdot 85397241 \cdot 56812934 \cdot 91245673 \cdot 72583416 .
\end{aligned}
$$

We close this section by comparing our theoretical results from earlier sections with the data from orders up to 9. Firstly, we expect from Lemma 7 to be able to construct pan-Hamiltonian squares of order $n$ from any perfect 1 -factorisation of $K_{n+1}$. Indeed, Lemma 8 shows that we may be able to construct more than one main class starting from a given 1-factorisation. However, all squares constructed in this way will have the symmetry that the square is isotopic to its row inverse. For $n=7$, Lemma 8 predicts (at least) two distinct squares, and the squares constructed there are easily seen to be isotopic to $\mathcal{C}_{7}$ and the square $A_{7}$ given in (3).

For $n=9$, Lemma 9 predicts at least one main class of pan-Hamiltonian square derived from a perfect 1-factorisation of $K_{10}$. In fact there is only one such 1-factorisation [14] and unlike the $n=7$ case, it produces a single main class. A representative of this class is $A_{9}$ as given in (4).

\section{$\S 7$. Other $N_{\infty}$ squares of order 9 .}

We saw in $\S 6$ that all main classes of $N_{\infty}$ squares of odd order less than 9 contain a pan-Hamiltonian square. In contrast, a minority of main classes of $N_{\infty}$ squares of order 9 have this property. We show this by looking at the subsquare structure of all $N_{2}$ squares of 
order 9, using a catalogue of the 1707 main classes of such square [10]. (The lists in $\S 6$ were also prepared from this catalogue.) Since a Latin square cannot have a proper subsquare of more than half its order and there are no $N_{2}$ squares of order 4 , we conclude that $N_{2}$ squares of order 9 cannot have proper subsquares except of order 3 . The breakdown of the 1707 main classes according to the number of order 3 subsquares appears in Table 1. For each main class the number of reduced squares in that class was calculated, and these totals are also given. (A Latin square of order $n$ is reduced if the entries in its first row and column are in the natural order $1,2,3, \ldots, n$. The total number of squares is a factor of $n !(n-1)$ ! larger than the number of reduced squares.)

\begin{tabular}{|c|c|c|}
\hline Order 3 subsquares & Main classes & Reduced squares \\
\hline 0 & 1589 & 28854493920 \\
1 & 46 & 550851840 \\
2 & 1 & 9797760 \\
3 & 15 & 123016320 \\
4 & 3 & 8164800 \\
6 & 5 & 18779040 \\
9 & 24 & 33653760 \\
10 & 9 & 46267200 \\
12 & 11 & 14152320 \\
18 & 3 & 453600 \\
36 & 1 & 840 \\
\hline Total & 1707 & 29659631400 \\
\hline
\end{tabular}

Table 1: Subsquares of $N_{2}$ squares of order 9 .

In particular we note that there are 1589 main classes of $N_{\infty}$ squares of order 9 , yet only 37 of these contain pan-Hamiltonian squares. In terms of reduced squares, there are $28854493920 N_{\infty}$ squares and 'only' 426746880 which are pan-Hamiltonian (or have a conjugate which is pan-Hamiltonian).

\section{$\S 8 . \quad$ References}

[1] L. D. Andersen, Factorizations of graphs, The CRC handbook of combinatorial designs (Eds: C. J. Colbourn and J. H. Dinitz), CRC Press, Boca Raton, FL, 1996, 653-667.

[2] L. D. Andersen and E. Mendelsohn, A direct construction for Latin squares without proper subsquares, Ann. Discrete Math. 15 (1982) 27-53.

[3] B. A. Anderson, A class of starter induced one-factorisations, Lecture notes in math. 406 (1974) 180-185. 
[4] A. E. Brouwer, Block designs, Handbook of combinatorics (Eds: R. L. Graham, M. Grötschel and L. Lovász), Elsevier, Amsterdam, 1995, 693-745.

[5] J. Dénes and A. D. Keedwell, Latin squares and their applications, Akadémiai Kiadó, Budapest, 1974.

[6] J. Dénes and A. D. Keedwell, Latin squares: New developments in the theory and applications, Annals Discrete Math. 46, North-Holland, Amsterdam, 1991.

[7] P. B. Gibbons and E. Mendelsohn, The existence of a subsquare free Latin square of side 12, SIAM J. Algebraic Discrete Methods 8 (1987) 93-99.

[8] K. Heinrich, Latin squares with no proper subsquares, J. Comb. Th. Ser. A 29 (1980) 346-353.

[9] A. J. W. Hilton and C. A. Rodger, Hamiltonian double Latin squares, preprint.

[10] B. D. McKay, private communication.

[11] H. W. Norton, The $7 \times 7$ squares, Ann. Eugenics 9 (1939) 269-307.

[12] A. Sade, An omission in Norton's list of $7 \times 7$ squares, Ann. Math. Statist. 22 (1951) 306-307.

[13] S. Stein, MR 82b05032, Mathematical reviews (1982) 486.

[14] W. D. Wallis, One-factorizations, Kluwer Academic, Dordrecht, Netherlands, 1997. 